\title{
Renormalization of the one-body off-diagonal Coulomb field in nuclei
}

\author{
N.Auerbach and O.K.Vorov \\ School of Physics and Astronomy, Tel Aviv University, Ramat Aviv, 69978 Tel Aviv, Israel
}

(June 17, 1997)

\begin{abstract}
Isospin violation effects in nuclei are investigated within a microscopic approach which takes into account the influence of the residual strong interaction on the Coulomb interaction. The renormalization of the off-diagonal Coulomb field acting within a nucleus, by the residual strong interaction is calculated analytically in a simplified RPA. From the expression for the suppression coefficient of the isospin violating part of the Coulomb field, the isospin violating spreading widths of isobaric analog states are derived. The resulting reduction of the width is in agreement with the data on the isospin symmetry restoration and with some earlier evaluations of the isospin violating spreading widths.
\end{abstract}

Typeset using REVTEX 
The off-diagonal part of the Coulomb potential in nuclei plays an important role in determining some properties of nuclear structure [1]- [11]. Many effects of isospin mixing including the widths of isobaric analog resonances are governed by off-diagonal matrix elements of the Coulomb one-body potential. It was realized [1]]- [6] that the off-diagonal one-body Coulomb matrix elements are strongly renormalized by the strong interaction in nuclei. This renormalization leads to reduced isospin mixing and the isospin symmetry is preserved to a better degree than what one would expect from unrenormalized Coulomb interactions. The method in which the renormalization was treated is by introducing collective effects into the radial motion in nuclei. The notion of the collective giant isovector monopole state (IVM) was introduced and its role as a mediator of many Coulomb mixing effects [1]- [7] was discussed twenty five years ago. The reduction in the Coulomb matrix elements is achieved by introducing a collective RPA upwards shift of the IVM and by a reduction of its strength due to the repulsive nature of the particle-hole interaction in the isovector channel.

The problem of isospin symmetry violation in complex nuclear states attracted attention for many years [1, $4,10,11,9,5]$ in the general context of theory of chaotic systems [12]. The case of isospin symmetry plays a special role in this respect because the very microscopic origin of its violation is well known and therefore constitutes a good example where one can test the theories of chaos in many-body systems.

A natural way to achieve this is to use an explicit realistic strong interaction that describes well nuclear properties and to employ RPA to evaluate the one-body Coulomb matrix elements. We are able to develop approximate analytical formulas which describe the renormalization of the off-diagonal Coulomb potential due to the strong nuclear force acting in nuclei. When realistic nuclear forces are used, the resulting Coulomb matrix elements are quenched and and are of the size that is capable to explain the experimentally observed reduction of the isospin violating spreading widths.

We start with the nuclear Hamiltonian $H$ written in the form

$$
H=H_{0}+V_{S}+U_{C},
$$


where the first term $H_{0}=\mathbf{p}^{2} / 2 m+U_{S}(r)$ is the single-particle Hamiltonian of nucleons moving in a single-particle strong potential $U_{S}(r)$. We will use the harmonic oscillator form $U_{S}=\frac{m \omega^{2}}{2} r^{2}$. The term $V_{S}$ is the residual two-body strong interaction. The one-body Coulomb potential is denoted by $U_{C}$ and we use the uniform density form:

$$
\begin{array}{r}
U_{C}=3 Z e^{2} /(2 R)\left(1-r^{2} /\left(3 R^{2}\right)\right), r \leq R \\
U_{C}=Z e^{2} / r, r>R
\end{array}
$$

where $R, a$, and $r$ are the nuclear radius, diffuseness, and the radial variable correspondingly.

We will be interested below in the RPA renormalization of the off-diagonal part of the Coulomb potential, $\tilde{U}_{C}$, defined as the rest of $U_{C}$ after subtraction of $\bar{U}_{C}$ which is diagonal in the representation in which the single-particle Hamiltonian $H_{0}$ is diagonal

$$
U_{C}=\tilde{U}_{C}+\bar{U}_{C}
$$

We now proceed [13,14 by defining an anti-Hermitean operator $\hat{A}$ and perform the following unitary transformation. The transformed Hamiltonian is:

$$
\begin{array}{r}
H^{*}=e^{\hat{A}} H e^{-\hat{A}}= \\
H_{0}+V_{S}+\tilde{U}_{C}+\bar{U}_{C}+\left[\hat{A}, H_{0}\right] \\
\\
+\left[\hat{A}, V_{S}\right]
\end{array}
$$

where we have used the decomposition (3) and neglected all terms above the first order in the Coulomb interaction. To obtain the effective one-particle off-diagonal Coulomb field, $\tilde{U}_{C}^{*}$, that results from the initial one, $\tilde{U}_{C}$, due to the resudual strong interaction effects, we should find the operator $\hat{A}$ in such a way that the single-particle off-diagonal Coulomb contribution in $e^{\hat{A}} H e^{-\hat{A}}$ will be cancelled. The last term in Eq.(四) is a two-body operator. We introduce the decomposition: $\left[\hat{A}, V_{S}\right] \equiv\left\langle\left[\hat{A}, V_{S}\right]\right\rangle+:\left[\hat{A}, V_{S}\right]$ :, where the first is a single-particle term obtained by averaging over the nucleon occupied states, and the second two-body term, : $\left[\hat{A}, V_{S}\right]:$, is the rest which yields zero under such averaging. 
We can compensate the one-body Coulomb type field in the Hamiltonian $H^{*}$, if we require the operator $\hat{A}$ to satisfy the equation

$$
\tilde{U}_{C}+\left[\hat{A}, H_{0}\right]+\left\langle\left[\hat{A}, V_{S}\right]\right\rangle=0
$$

Then, the transformed Hamiltonian takes the form

$$
\tilde{H}=H_{0}+V_{S}+\bar{U}_{C}+:\left[\hat{A}, V_{S}\right]:
$$

where no single-particle off-diagonal Coulomb potential is present. In this case, the effective off-diagonal Coulomb potential field can be found from the equation

$$
\tilde{U}_{C}^{*}=-\left[\hat{A}, H^{0}\right]
$$

where the effects of the renormalization of the $\tilde{U}_{C}$ are incorporated into the operator $A$ [13]. In the language of the present approach, the operator $\hat{A}$ in Eq.(四) creates a small distortion of the nuclear density matrix when acting on the density matrix which contains no effects due to the residual interaction $V_{S}$. Equations (5),(7) can be seen to be equivalent to the main equation of the Theory of Finite Fermi Systems [15]

$$
\tilde{\mathcal{F}}_{i k}^{*}=\tilde{\mathcal{F}}_{i k}+\sum_{\alpha \beta}\left(V_{S}\right)_{\beta \alpha, i k} \frac{n_{\beta}-n_{\alpha}}{\varepsilon_{\beta}-\varepsilon_{\alpha}-\omega} \tilde{\mathcal{F}}_{i k}^{*}
$$

which relates an initial off-diagonal field $\tilde{\mathcal{F}}$ to its effective field $\tilde{\mathcal{F}}^{*}$, obtained via summation of the series of RPA diagrams involving the residual interaction and the particle-hole propagator. $\mathcal{A}$ [15]. In Eq.(8), indices refer to the single-particle nucleon states which label the matrix elements of the fields $\tilde{\mathcal{F}}, \tilde{\mathcal{F}}^{*}$, the interaction $V_{S}$ and the single-particle occupation probabilities $n_{i}$ of the states with the energies $\varepsilon_{i}$. In our case, the external (Coulomb) field is static, so $\omega=0$. Indeed, substituting Eq.(17) into Eq.(15) and collecting the terms one obtains an equation analogous to Eq.(8) with $\tilde{\mathcal{F}}^{*}$ and $\tilde{\mathcal{F}}$ replaced by $\tilde{U}^{*}$ and $\tilde{U}$. Equations (5),(可) provide a convenient way to obtain analytical results for the renormalization of the potential in the approximation which we develop below. 
Up to now, we did not specify the explicit form of the residual strong interaction. To obtain results for the renormalization according to Eqs.(55,0), one needs to specify $V_{S}$. We use below the Landau-Migdal parametrization [15], [16] of the strong interaction $V_{S}$,

$$
V_{S}\left(\mathbf{r}, \mathbf{r}^{\prime}\right)=C \delta\left(\mathbf{r}-\mathbf{r}^{\prime}\right)\left[f+f^{\prime} \tau \tau^{\prime}+g \sigma \sigma^{\prime}+g^{\prime} \tau \tau^{\prime} \sigma \sigma^{\prime}\right]
$$

which has been widely used for heavy nuclei (see [15], [16]). One therefore considers the form (9) as a reliable parametrization of $V_{S}$ that reproduces correctly the main properties of actual residual interaction. The strengh constants $f, f^{\prime}, g$, and $g^{\prime}$ depend on the nuclear density. The relevant part of the interaction Eq.(9), $V_{r}$, that is responsible for the renormalization of the one-body off-diagonal Coulomb field is given by the first two terms of Eq.(9) whose explicit form reads as

$$
\begin{array}{r}
V_{r}\left(\mathbf{r}, \mathbf{r}^{\prime}\right)=C \delta\left(\mathbf{r}-\mathbf{r}^{\prime}\right)\left[f_{\text {in }}-\left(f_{e x}-f_{i n}\right)\left(\rho(r)-\rho_{0}\right) \rho_{0}^{-1}+\right. \\
\left.+\left(f_{\text {in }}^{\prime}-\left(f_{\text {ex }}^{\prime}-f_{i n}^{\prime}\right)\left(\rho(r)-\rho_{0}\right) \rho_{0}^{-1}\right)\left(\tau \tau^{\prime}\right)\right] .
\end{array}
$$

Here, $\rho(r)$ is the nuclear density, and $\rho_{0}$ denotes its value in the center of nucleus. Here $C=300 \mathrm{MeV} \mathrm{fm}^{3}$ is the universal Migdal constant. The values of the strength parameters are [15]: $f_{e x}=-1.95, f_{i n}=-0.075 f_{e x}^{\prime}=0.05 f_{i n}^{\prime}=0.675$.

From symmetry considerations, the operator $\hat{A}$ can be taken in the form:

$$
\hat{A}=x_{p}\left(\left(\vec{\nabla}_{p} \vec{r}_{p}\right)+\left(\vec{r}_{p} \vec{\nabla}_{p}\right)\right)+x_{n}\left(\left(\vec{\nabla}_{n} \vec{r}_{n}\right)+\left(\vec{r}_{n} \vec{\nabla}_{n}\right)\right),
$$

to match equation (5) with some constants $x_{p}, x_{n}$ to be determined.

In the expression for $\left\langle\left[A, V_{S}\right]\right\rangle$ we have, after some calculations,

$$
\left\langle\left[A, V_{S}\right]\right\rangle=2 \frac{C \rho_{0}}{\left|U_{0}\right|}\left(S_{p}-R_{p} r_{p}^{2}+S_{n}-R_{n} r_{n}^{2}\right) .
$$

The off-diagonal part of Eq.(121) which is to be substituted into Eq.(河) is given by the offdiagonal parts of the second and the fourth terms in (12) involving the off-diagonal terms of $r_{p}^{2}$ and $r_{n}^{2}$ (denoted by $\tilde{r}_{p}^{2}$ and $\tilde{r}_{n}^{2}$ ). The following combinations are introduced: 


$$
\begin{array}{r}
S_{p}=3 U_{0}\left(x_{p} F_{p p} \frac{Z}{A}+x_{n} F_{p n} \frac{N}{A}-x_{p} F_{p p}^{\prime} \frac{Z}{A}-x_{n} F_{p n}^{\prime} \frac{N}{A}\right) \\
R_{p}=m \omega^{2}\left[\frac{5}{2}\left(x_{p} F_{p p} \frac{Z}{A}+x_{n} F_{p n} \frac{N}{A}\right)-\gamma\left(x_{p} F_{p p}^{\prime} \frac{Z}{A}+x_{n} F_{p n}^{\prime} \frac{N}{A}\right)\right] \\
S_{n}=3 U_{0}\left(x_{p} F_{n p} \frac{Z}{A}+x_{n} F_{n n} \frac{N}{A}-x_{p} F_{n p}^{\prime} \frac{Z}{A}-x_{n} F_{n n}^{\prime} \frac{N}{A}\right) \\
R_{n}=m \omega^{2}\left[\frac{5}{2}\left(x_{p} F_{n p} \frac{Z}{A}+x_{n} F_{n n} \frac{N}{A}\right)-\gamma\left(x_{p} F_{n p}^{\prime} \frac{Z}{A}+x_{n} F_{n n}^{\prime} \frac{N}{A}\right)\right]
\end{array}
$$

The new constants are defined as follows

$$
\begin{aligned}
& F_{p p}=F_{n n}=f_{e x}+f_{i n}^{\prime}, \quad F_{p p}^{\prime}=F_{n n}^{\prime}=-\left(f_{e x}-f_{i n}+f_{e x}^{\prime}-f_{i n}^{\prime}\right), \\
& F_{p n}=F_{n p}=f_{e x}-f_{i n}^{\prime}, \quad F_{p n}^{\prime}=F_{n p}^{\prime}=-\left(f_{e x}-f_{i n}-f_{e x}^{\prime}+f_{i n}^{\prime}\right) .
\end{aligned}
$$

Now, substituting Eq.(12) into Eq.(可) and accouning for (13),(14) we obtain, after separating the similar operator structures, the following system of equations:

$$
\left(\begin{array}{cc}
1-\frac{Z}{2 A} \frac{C \rho_{0}}{\left|U_{0}\right|}\left(\frac{5}{2} F_{p p}-\gamma F_{p p}^{\prime}\right) & -\frac{N}{2 A} \frac{C \rho_{0}}{\left|U_{0}\right|}\left(\frac{5}{2} F_{p n}-\gamma F_{p n}^{\prime}\right) \\
-\frac{Z}{2 A} \frac{C \rho_{0}}{\left|U_{0}\right|}\left(\frac{5}{2} F_{n p}-\gamma F_{n p}^{\prime}\right) & 1-\frac{N}{2 A} \frac{C \rho_{0}}{\left|U_{0}\right|}\left(\frac{5}{2} F_{n n}-\gamma F_{n n}^{\prime}\right)
\end{array}\right)\left(\begin{array}{c}
x_{p} \\
x_{n}
\end{array}\right)=\left(\begin{array}{c}
\frac{q_{p}}{4 m \omega^{2}} \\
0
\end{array}\right)
$$

The solution for this simple system of linear equations is found inverting the matrix in Eq.(15); the value of the determinant is given by the formula

$$
\begin{aligned}
D=\left[1-\frac{Z}{2 A} \frac{C \rho_{0}}{\left|U_{0}\right|}\left(\frac{5}{2} F_{p p}-\gamma F_{p p}^{\prime}\right)\right] & {\left[1-\frac{N}{2 A} \frac{C \rho_{0}}{\left|U_{0}\right|}\left(\frac{5}{2} F_{n n}-\gamma F_{n n}^{\prime}\right)\right]-} \\
& -\frac{Z N}{4 A^{2}}\left(\frac{C \rho_{0}}{\left|U_{0}\right|}\right)^{2}\left(\frac{5}{2} F_{p n}-\gamma F_{p n}^{\prime}\right)^{2}
\end{aligned}
$$

Instead of the initial off-diagonal Coulomb field

$$
\tilde{U}_{C}^{(p)}=-q \tilde{r}_{p}^{2}, \quad \tilde{U}_{C}^{(n)}=0
$$

using Eqs.(7),(11) and finding $x_{p}, x_{n}$ from the system of equation (15), we obtain now proton and neutron effective (renormalized) fields which are given by the expressions:

$$
\begin{gathered}
\tilde{U}_{C}^{*(p)}=-\frac{1-\frac{N}{2 A} \frac{C \rho_{0}}{\left|U_{0}\right|}\left(\frac{5}{2} F_{n n}-\gamma F_{n n}^{\prime}\right)}{D} q \tilde{r}_{p}^{2}, \\
\tilde{U}_{C}^{*(n)}=-\frac{\frac{Z}{2 A} \frac{C \rho_{0}}{\left|U_{0}\right|}\left(\frac{5}{2} F_{n p}-\gamma F_{n p}^{\prime}\right)}{D} q \tilde{r}_{n}^{2},
\end{gathered}
$$


(the asterisk marks the renormalized quantities). The effective field that acts on neutrons is non zero, it contains now the contributions of the proton-neutron component of the residual strong interaction. The sign of this contribution is opposite to that of the proton field.

For the renormalization of the isovector part of the off-diagonal Coulomb field, $\tilde{U}_{c}^{*(i)} \equiv$ $\tilde{U}_{C}^{*(p)}-\tilde{U}_{C}^{*(n)}$, that can be written as

$$
\tilde{U}_{c}^{*(i)}=\left(q_{p}^{*}-q_{n}^{*}\right) \tilde{r}^{2} \equiv \frac{1}{S}\left(q \tilde{r}^{2}\right)
$$

we obtain from Eqs.(17, 18) the following expression:

$$
\begin{gathered}
\frac{1}{S}=\frac{q_{p}^{*}-q_{n}^{*}}{q}= \\
=\frac{1-\frac{N}{2 A} \frac{C \rho_{0}}{\left|U_{0}\right|}\left(\frac{5}{2} F_{n n}-\gamma F_{n n}^{\prime}\right)-\frac{Z}{2 A} \frac{C \rho_{0}}{\left|U_{0}\right|}\left(\frac{5}{2} F_{n p}-\gamma F_{n p}^{\prime}\right)}{\left[1-\frac{Z}{2 A} \frac{C \rho_{0}}{\left|U_{0}\right|}\left(\frac{5}{2} F_{p p}-\gamma F_{p p}^{\prime}\right)\right]\left[1-\frac{N}{2 A} \frac{C \rho_{0}}{\left|U_{0}\right|}\left(\frac{5}{2} F_{n n}-\gamma F_{n n}^{\prime}\right)\right]-\frac{Z N}{4 A^{2}}\left(\frac{C \rho_{0}}{\left|U_{0}\right|}\right)^{2}\left(\frac{5}{2} F_{p n}-\gamma F_{p n}^{\prime}\right)^{2}}
\end{gathered}
$$

This analytical result gives the value of the suppression factor $S$ of the isospin violating part of the Coulomb field as a function of the nuclear charge and mass, and the interaction constants. A simple numerical evaluation of Eq.(19) for the case $N \simeq Z$ and the values of the interaction strengths presented above (see Eq.(10)) gives the following value of the suppression factor $S$

$$
S=1.8 \pm 0.4
$$

where the uncertainty is mainly due to that in the quantity $\gamma$. The obtained value of the reduction factor squared $S^{2} \simeq 3.4 \pm 1.4$ can be compared to the suppression factor that results from comparison of the experimental spreading widths of the IAS resonances with the values calculated using the unrenormalized Coulomb potential:

$$
S_{\text {exp }}^{2} \sim 3-4
$$

Therefore, the present theoretical result for the renormalization of the off-diagonal isovector Coulomb field can be considered as a microscopic theoretical explanation of reduced isospin symmetry breaking in nuclei.

The utilization of the above effective off-diagonal Coulomb potential is limited to the inside of the nucleus and to the states localized inside. We should keep in mind that the 
reduction derived above is applicable only to the $r^{2}$ part of Eq.(2) and is not applicable for the outside $(r>R)$ part that goes as $\frac{1}{r}$.

The way in which our present result could be applied is illustrated in the following two examples:

A. Isospin mixing in ground states. In order to calculate isospin mixing we can use the potential $\tilde{U}_{c}^{*}$ in the expression [1]:

$$
\left(\varepsilon^{*}\right)^{2}=\sum_{n l j} \frac{\left|\left\langle n l j\left|\tilde{U}_{c}^{*(i)}\right| n+1 l j\right\rangle\right|^{2}}{\left(E_{n}-E_{n+1}\right)^{2}}
$$

where $|n l j\rangle$ are single-paticle states and $|n+1 l j\rangle$ the corresponding radially excited s.p. states. The denominator is the energy difference between the unperturbed single-particle states. The collective effects due to RPA correlations are taken into account in $\tilde{U}_{c}^{*(i)}$. We find that

$$
\left(\varepsilon^{*}\right)^{2}=\frac{\varepsilon^{2}}{S^{2}}
$$

where $\varepsilon$ is the amount of isospin mixing in the single-particle model without the suppression effect calculated in the present work. We see that our model predicts a reduction in isospin mixing of about a factor $S^{2} \sim 4$ compared to the pure single-particle model in which the renormalization of the off-diagonal Coulomb field due to the strong interaction is not taken into acount.

B.Spreading width of isobaric analog resonances. In the single-particle approximation the spreading width is given by [1, [4, 6 ]

$$
\Gamma_{A}^{\downarrow}=-\operatorname{Im}\left\langle A\left|\tilde{U}_{c} G_{P}^{+} \tilde{U}_{c}\right| A\right\rangle
$$

where $|A\rangle$ is the analog state, and $G_{P}^{+}$is the optical potential model Green's function. As already discussed this kind of calculation overstimates the spreading widths of isobaric analog resonances by factors close to 5 for standard optical potentials [1]. If we replace $\tilde{U}_{c}$ by $\tilde{U}_{c}^{*(i)}$ we find that the spreading widths are much closer to the experimental ones and close to the results obtained in Ref. [6] where the collectivity of the IVM is taken into account. 
We should stress that this reduction applies only to the spreading widths and not to the decay (escape) widths $\Gamma_{A}^{\uparrow}$. The latter involves continuum wave-function which are not localized inside the nucleus and therefore $\Gamma_{A}^{\uparrow}$ depends on the $1 / r$ part of $U_{c}$. As mentioned above the reduction we find does not apply to $1 / r$ and therefore it affects the escape width much less, in agreement with the calculations in Ref. [3].

Some of the reduction of isospin mixing occurs already in the Hartee-Fock (HF) approximation 4 . The attractive proton-neutron force couples the proton and neutron distributions reducing the difference in the two caused by the Coulomb force. In an unrestricted HF calculation that allows for deformations and for charge-exchange modes one obtaines in the wave fuctions components that can be expressed as a particles coupled to the various RPA states (including the IVM) of the core. Thus in such extended HF calculation effects of the RPA will be included. However the use of such unrestricted RPA is not practical and the use of such scheme for the quantitites calculated in this work is limited for the following reasons. (a) In order to incorporate correctly isovector effects in the HF one must include charge-exchange excitations, which is a difficult task. (b) The HF calculation in an odd A system involves additional approximations. (c) The usual HF approach in nuclei with a neutron excess introduces spurious isospin mixing and the symmetry potential acts as an isospin breaking term [4]. (d) The HF approximation cannot be used for unbound states.

To summarize, we have developed here an approximate analytical approach to study the role played by the residual strong interaction in reducing the Coulomb matrix elements violating isospin symmetry in nuclear states. We calculated for the first time analytically the RPA renormalization of the off-diagonal Coulomb potential starting from the nuclear Hamiltonian with the residual two-body strong nuclear forces. When realistic nuclear forces (Landau-Migdal parametrization) is used our approach produces quenched Coulomb matrix elements that are capable of explaining the experimental isospin violating spreading widths.

This work is supported by the Grant for Basic Research of the Israeli Academy of Science. 


\section{REFERENCES}

[1] N.Auerbach, J. Hüfner, A.K. Kerman and C.M. Shakin, Rev. Mod. Phys., 44, 48 (1972).

[2] A.Z.Mekjan, Phys. Rev. Lett., 25, 888 (1970).

[3] N.Auerbach and G.Bertsch, Phys. Lett. 43B, 175 (1973).

[4] N.Auerbach, Phys. Rep. C98, 273 (1983).

[5] N.Auerbach and N. van Giai, Phys. Lett. B72, 289 (1978).

[6] N.Auerbach, Phys. Lett. 44B, 241 (1973).

[7] A.Bohr and B.Mottelson, Nuclear Structure (Benjamin, New York, 1969), Vol. 1.

[8] H.L.Harney, A.Richter and H.A.Weidenmüller, Rev. Mod. Phys., 58, 607 (1986).

[9] G.E.Mitchell, E.G.Bilpuch, P.M.Endt, and J.F. Shriner, Phys.Rev.Lett., 61, 1473 (1988).

[10] T.Guhr and H.A. Weidenmüller, Ann.Phys. (N.Y.), 199, 412 (1990).

[11] H.A. Weidenmüller, Nucl.Phys. A552, 293c (1991).

[12] T.A.Brody, J.Flores, J.B.french, P.A.Mello, A.Pandey, and S.S.M.Wong, Rev. Mod.Phys. 53, 385 (1981).

[13] V.V.Flambaum and O.K.Vorov, Phys. Rev. C51, 1521 (1995).

[14] V.V.Flambaum and O.K.Vorov, Phys.Rev. C49, 1827 (1994).

[15] A.B.Migdal, Theory of Finite Fermi Systems and Applications to atomic Nuclei (John Wiley \& Sons, New York, 1967).

[16] G.E.Brown, Rev.Mod.Phys., 43,1 1971); V.Klemt, S.A.Moszkowski, and J.Speth, Phys.Rev. C14, 302 (1976); J.Speth, E.Werner, and W.Wild, Phys.Rep. 33, No.3, 127(1977); G.Bertsch, D.Cha, and H.Toki, Phys.Rev. C24, 533 (1981); V.A.Khodel and E.E.Sapershtein, Phys.Rep. 92, 183 (1982), K. Goeke, and J.Speth, Annu. Rev. Nucl. 
Part. Sci. 32, 65 (1982); F.Osterfeld, Rev.Mod.Phys., 64, 491 (1992), and references therein. 